\title{
New connected sums with positive Ricci curvature
}

\author{
David J. Wraith
}

Received: 16 October 2006 / Accepted: 15 February 2007 / Published online: 30 March 2007

(C) Springer Science + Business Media B.V. 2007

\begin{abstract}
We construct a new infinite family of Ricci positive manifolds, generalising a well-known result of Sha and Yang.
\end{abstract}

Keywords Positive Ricci curvature $\cdot$ Connected sums $\cdot$ Surgery

Mathematics Subject Classification (2000) 53C20

\section{Introduction}

One would ultimately like to understand which complete Riemannian manifolds admit metrics of everywhere positive Ricci curvature. However, this is a very difficult proposition and out of range at present. In fact very little is known about the topological consequences of positive Ricci curvature, and examples of positive Ricci curvature manifolds are relatively thin on the ground. The aim of this paper is to present a construction technique which yields infinitely many new examples of such manifolds.

In [5], Sha and Yang showed that connected sums of the form

$$
\sharp_{i=1}^{k} S^{n} \times S^{m}
$$

admit metrics of positive Ricci curvature for $n, m \geq 2$. Note that connected sums between Ricci positive manifolds will not in general support a metric of positive Ricci curvature, as can be seen from Myers' Theorem [4; p. 245]. (This is in contrast to the situation for positive scalar curvature, where connected sums of positive scalar curvature manifolds admit positive scalar curvature metrics provided the dimension is at least three [3].) The result of Sha and Yang is a consequence of the fact that

Communicated by I. Taimanov (Novosibirsk).

D. J. Wraith $(\varangle)$

Department of Mathematics, NUI Maynooth,

Maynooth, Ireland

e-mail: David.Wraith@nuim.ie 
their manifolds admit an alternative description in terms of performing surgeries (of dimension greater than zero) on a fixed Ricci positive manifold.

This paper is also concerned with connected sums between products of spheres. However, the factor spheres are now allowed to have different dimensions to those in other products. The main aim of this paper is to establish the following result.

Theorem A The manifold

$$
\left(S^{n_{1}} \times S^{m_{1}}\right) \sharp\left(S^{n_{2}} \times S^{m_{2}}\right) \sharp \ldots . . \sharp\left(S^{n_{k}} \times S^{m_{k}}\right)
$$

admits a metric of positive Ricci curvature for any $n_{i}, m_{i} \geq 3$ such that $n_{i}+m_{i}=n_{j}+m_{j}$ for all $1 \leq i, j \leq k$.

The simplest new example occurs in dimension 10, namely $\left(S^{3} \times S^{7}\right) \sharp\left(S^{4} \times S^{6}\right) \sharp\left(S^{5} \times S^{5}\right)$. The essential difficulty in establishing Theorem $\mathrm{A}$ is that different products of spheres display different symmetries.

Note this this result is not sharp. Sha and Yang show that connected sums of the form $\left(S^{2} \times S^{n}\right) \sharp \cdots \sharp\left(S^{2} \times S^{n}\right)$ admit positive Ricci curvature metrics for $n \geq 2$. As explained in Sect. 4 , so do manifolds such as $\left(S^{2} \times S^{4}\right) \sharp\left(S^{3} \times S^{3}\right)$. It would be interesting to know whether Theorem A would still be true if we were to allow any number of $S^{2}$ factor spheres in our products.

It is not difficult to extend Theorem A to more general situations. We present two such extensions.

Theorem B Suppose that $M$ has the structure of an $S^{m}$-bundle over a compact base $X^{n}$, with a Lie structural group. If $X$ admits a metric of positive Ricci curvature and $m>n \geq 3$, then any connected sum

$$
M \sharp\left(S^{n_{1}} \times S^{m_{1}}\right) \sharp \cdots \sharp\left(S^{n_{k}} \times S^{m_{k}}\right)
$$

admits a metric of positive Ricci curvature for $n_{i}, m_{i} \geq 3$ and $n_{i}+m_{i}=n+m$ for all $i$.

Note that if we allow $m \leq n$ in Theorem $\mathrm{B}$, this will restrict the possible factor sphere dimensions.

Theorem C Consider $M$ as in Theorem B, with the additional requirement that $m \geq$ $n+5$. Let $E_{1}, \ldots, E_{k}$ be sphere bundles with base $S^{d_{i}}$, fibre $S^{n+m-d_{i}}$, and structural group $S O\left(n+m-d_{i}+1\right)$, where $m-1 \geq d_{i} \geq n+4$ for $i=1, \ldots, k$. Then

$$
M \sharp E_{1} \sharp \ldots . \sharp E_{k}
$$

admits a metric with positive Ricci curvature.

The results in this paper are produced using surgery. As explained in Sect. 2, $\left(S^{n} \times S^{m}\right) \sharp\left(S^{p+1} \times S^{n+m-p-1}\right)$ can be created from $S^{n} \times S^{m}$ by performing a surgery on a suitably embedded $S^{p}$. We choose to embed this $S^{p}$ in the $S^{m}$ factor of the product. Similarly for further connected sums. To the best of the author's knowledge, these are the first examples of Ricci positive manifolds to be constructed by performing surgeries of different dimensions on the starting manifold.

To ensure our constructions admit positive Ricci curvature metrics, we work explicitly with metrics of cohomogeneity two.

The rest of this paper is laid out as follows. In Sect. 2 we sketch the topology behind our examples. In Sect. 3 we give a brief overview of the metric construction process. Some existing Ricci positive surgery results are noted in Sect. 4, and in Sect. 5 we list the relevant Ricci curvature formulas. The main metric construction is described in Sect. 6, and the main theorems are proved in Sect. 7. 


\section{Constructing the connected sums}

We begin by describing the process of surgery. Consider a smooth embedding

$$
\iota: S^{n} \longrightarrow M^{n+m} \text {. }
$$

Assume the normal bundle of $\iota\left(S^{n}\right)$ is trivial. This means we can extend $\iota$ to an embedding

$$
e: S^{n} \times D^{m} \longrightarrow M
$$

Performing surgery involves cutting something out - the interior of the image of the embedding $e$-and stitching something in - a copy of $D^{n+1} \times S^{m-1}$. (Note that the boundary of $S^{n} \times D^{m}=S^{n} \times S^{m-1}=$ boundary of $D^{n+1} \times S^{m-1}$.) The dimension of such a surgery is $n$, and the codimension is $m$.

On the assumption that $p<m$, the manifold $\left(S^{n} \times S^{m}\right) \sharp\left(S^{p+1} \times S^{n+m-p-1}\right)$ can be constructed from $S^{n} \times S^{m}$ by performing a single $p$-surgery. Specifically, we perform surgery on $\{*\} \times S^{p} \subset S^{n} \times S^{m}$, which is contractible within $S^{n} \times S^{m}$. More generally,

$$
M^{m_{\sharp}}\left(S^{p+1} \times S^{m-p-1}\right)
$$

can be constructed by performing a surgery on a contractibly embedded $p$-sphere in $M$. To see this, first note that

$$
M \cong M \sharp S^{m} .
$$

Now

$$
S^{m}=\left(S^{p} \times D^{m-p}\right) \cup\left(D^{p+1} \times S^{m-p-1}\right) \quad\left[=\partial\left(D^{p+1} \times D^{m-p}\right)\right] .
$$

Therefore

$$
M \cong M \sharp\left(D^{p+1} \times S^{m-p-1}\right) \cup\left(S^{p} \times D^{m-p}\right) .
$$

The result of performing the obvious surgery is

$$
\begin{gathered}
M \sharp\left(D^{p+1} \times S^{m-p-1}\right) \cup\left(D^{p+1} \times S^{m-p-1}\right) \\
=M \sharp\left(S^{p+1} \times S^{m-p-1}\right) .
\end{gathered}
$$

Our examples will be produced by performing a sequence of surgeries on spheres embedded in distinct copies of $S^{m}$ within the product $S^{n} \times S^{m}$.

\section{A metric overview}

We will begin with a product of round metrics on our starting manifold $S^{n} \times S^{m}$. Let us assume that on $S^{n}$ the round metric has unit radius, and on $S^{m}$ the radius will be small and fixed at a later point in the construction.

We first decide how many surgeries (connected sums) we wish to perform. We then remove that many disjoint copies of $D^{n} \times S^{m}$ from our product. We assume that all disks are geodesic disks of the same radius. The number of surgeries will then determine an upper bound for the disk radius. 
Our idea is then to replace these by inserting a 'lid' into each of the holes. Each lid is also a copy of $D^{n} \times S^{m}$, so topologically this achieves nothing. However, metrically these lids will be somewhat different to those removed. Each lid will have non-negative Ricci curvature, and have a metric near the boundary, which can be smoothly joined with the ambient metric within positive Ricci curvature. Moreover, the lid metric will be such as to allow a Ricci positive surgery of the desired dimension at its centre. To perform this surgery we use the result of Sha-Yang, which will be outlined in Sect. 4 below. The fact that some Ricci curvatures are zero is not a problem, because at many points all Ricci curvatures are positive. Under these circumstances a result of Ehrlich [2] guarantees the existence of a positive Ricci curvature metric on the whole manifold. The precise metric details of the lid are described in Sect. 6.

\section{Ricci positive surgery}

Consider the following situation. Suppose we are given a Riemannian manifold $M^{p+d}$ having positive Ricci curvature and an isometric embedding $\iota: S^{p}(\rho) \times D_{R}^{d}(N) \longrightarrow M$ where $D_{R}^{d}(N)$ denotes a geodesic ball of radius $R$ in the $d$-sphere with the round metric of radius $N$. $S^{p}(\rho)$ is the $p$-sphere with the round metric of radius $\rho$. We can regard $\iota$ as a trivialisation of the normal bundle of $\iota\left(S^{p} \times\{0\}\right)$.

Theorem (Sha, Yang [5]). Let $\hat{M}$ be the result of performing surgery on $\iota\left(S^{p} \times\{0\}\right)$ using the trivialisation $\iota$, and assume $p \geq 1, d \geq 3$. Then there exists $\kappa\left(p, d, R N^{-1}\right)>0$ such that if $\frac{\rho}{N}<\kappa$ then $\hat{M}$ can be equipped with a Ricci positive metric.

This result will be a vital ingredient in the proof of Theorem A.

As mentioned in Sect. 1, Sha and Yang used this result to establish the existence of Ricci positive metrics on $\sharp_{i=1}^{k} S^{n} \times S^{m}$ for $n, m \geq 2$. It is not difficult to adapt the result to produce different examples.

Consider a product of spheres $S^{n} \times S^{m}$, which is equipped with a product metric consisting of a round metric on $S^{n}$, and on $S^{m}$ a metric of the form

$$
\mathrm{d} t^{2}+f^{2}(t) \mathrm{d} s_{p}^{2}+h^{2}(t) \mathrm{d} s_{m-p-1}^{2}
$$

for $t \in\left[0, \frac{\pi}{2}\right]$. Here we need to assume $n \geq 2$ and $m-2 \geq p \geq 1$. Set $h(t)=\sin t$. Suppose $f(t)=\cos t$, for $t \geq \delta^{\prime}$, some $\delta^{\prime}$ close to $\frac{\pi}{2} ; f(t)=\delta$, a small constant, for $t \in\left[0, \frac{\pi}{4}\right]$; and that $f^{\prime \prime}(t) \leq 0$ for all $t$. Suppose we want to perform surgery on the copy of $S^{p}$ corresponding to $t=0$. Topologically, a neighbourhood of this sphere is $S^{p} \times D^{n+m-p}$, and metrically we have a product metric between a round metric of radius $\delta$ on $S^{p}$ and a Ricci positive metric on $D^{n+m-p}$. As noted in [5; p. 134], we can locally deform the metric on $D^{n+m-p}$ within Ricci positivity so that it is round of some radius in a neighbourhood of the origin. It is clear that $f(t)$ and in particular the constant $\delta$ can be chosen independently of the metric on $D^{n+m-p}$. Therefore, by choosing $\delta$ small enough, Sha-Yang guarantees the existence of a Ricci positive metric on the manifold obtained by performing surgery on this $S^{p}$. Thus we see that $\left(S^{n} \times S^{m}\right) \sharp\left(S^{p+1} \times S^{n+m-p-1}\right)$ admits a Ricci positive metric.

Observe also that where our normal disk is round, we can in fact perform multiple Ricci positive $p$-surgeries, by choosing $\delta$ smaller if necessary. Indeed, with a little more work it can be shown that

$$
\left(\sharp_{i=1}^{k} S^{n} \times S^{m}\right) \sharp\left(\sharp_{j=1}^{l} S^{p+1} \times S^{n+m-p-1}\right)
$$

admits a Ricci positive metric, as pointed out in [5].

黑 Springer 
It is difficult to see how to adapt this approach to deal with connected sums between products of spheres where we have products of three or more different types. The symmetry of the metric described above seems to obstruct further surgeries of differing dimensions.

In our construction below, we produce a new method which deals with this problem. The underlying reason why our method is able to succeed in this situation is that we work explicitly with metrics of cohomogeneity two. The metrics constructed by Sha and Yang only have cohomogeneity one, and so are less flexible.

\section{The curvature formulas}

In our metric construction of the lid, we will consider a metric of the form

$$
\mathrm{d} s^{2}+\mathrm{d} t^{2}+f^{2}(s, t) \mathrm{d} s_{p}^{2}+g^{2}(t) \mathrm{d} s_{q}^{2}+h^{2}(s) \mathrm{d} s_{n-1}^{2}
$$

on

$$
[0, \infty) \times\left[0, \frac{\pi}{2}\right] \times S^{p} \times S^{q} \times S^{n-1} .
$$

The Ricci curvature formulas for this metric are easily calculated, for example using [1; p. 266], and we omit the details.

With $f, g$ and $h$ satisfying suitable boundary conditions (see Sect. 6), we can regard (*) as a metric on $D^{n} \times S^{p+q+1}$.

In the following, let $\left\{X_{i}\right\}$ denote a local orthonormal frame field on $S^{p}$, let $\left\{V_{j}\right\}$ be an orthonormal frame field for $S^{q}$, and let $\left\{W_{k}\right\}$ denote an orthonormal frame field for $S^{n-1}$. (Here, orthonormal means with respect to the above metric.)

$$
\begin{aligned}
\operatorname{Ric}\left(\frac{\partial}{\partial s}, \frac{\partial}{\partial s}\right) & =-p \frac{f_{s s}}{f}-(n-1) \frac{h_{s s}}{h} . \\
\operatorname{Ric}\left(\frac{\partial}{\partial t}, \frac{\partial}{\partial t}\right) & =-p \frac{f_{t t}}{f}-q \frac{g_{t t}}{g} . \\
\operatorname{Ric}\left(\frac{\partial}{\partial s}, \frac{\partial}{\partial t}\right) & =-p \frac{f_{s t}}{f} . \\
\operatorname{Ric}\left(X_{i}, X_{i}\right) & =-\frac{f_{s s}+f_{t t}}{f}+\frac{p-1}{f^{2}}\left(1-f_{s}^{2}-f_{t}^{2}\right)-q \frac{f_{t} g_{t}}{f g}-(n-1) \frac{f_{s} h_{s}}{f h} . \\
\operatorname{Ric}\left(V_{j}, V_{j}\right) & =-\frac{g_{s s}+g_{t t}}{g}+\frac{q-1}{g^{2}}\left(1-g_{s}^{2}-g_{t}^{2}\right)-p \frac{f_{t} g_{t}}{f g} . \\
\operatorname{Ric}\left(W_{k}, W_{k}\right) & =-\frac{h_{s s}}{h}+(n-2) \frac{1-h_{s}^{2}}{h^{2}}-p \frac{f_{s} h_{s}}{f h} .
\end{aligned}
$$

All other Ricci curvature terms vanish.

\section{Constructing the 'lid'}

From now on we will make the following dimensional assumptions:

$$
\begin{aligned}
& n \geq 3, \\
& p \geq 2, \\
& q \geq 1 .
\end{aligned}
$$


Topologically, the lid is a product $D^{n} \times S^{p+q+1}$, with $p+q+1=m$. Geometrically, we assume a metric of the form $(*)$. We will supppose that we wish to perform surgery on a copy of $S^{p} \subset S^{m}$ in the centre of the lid. It follows from our dimensional assumptions that the surgeries we aim to perform have dimension at least 2 and at most $m-2$.

In this section we show how to choose the functions $f, g$ and $h$. We begin by detailing the boundary conditions we require these functions to satisfy.

We want the $\mathrm{d} s^{2}+h^{2}(s) \mathrm{d} s_{n-1}^{2}$ part of the metric $(*)$ to be our metric on $D^{n}$. For this to be a well-defined smooth metric at the origin of $D^{n}$, we require $h(s)$ to be everywhere smooth and odd at $s=0$. For $h$ to be odd at $s=0$ we demand:

$$
\begin{gathered}
h(0)=0 ; \\
h^{(2 i)}(0)=0
\end{gathered}
$$

for every integer $i \geq 1$. These conditions are necessary but not sufficient for smoothness at the origin of $D^{n}$. For sufficiency (see [4; p. 13]) we also require:

$$
h^{\prime}(0)=1 \text {. }
$$

We want $\mathrm{d} t^{2}+f^{2}(s, t) \mathrm{d} s_{p}^{2}+g^{2}(t) \mathrm{d} s_{q}^{2}$ to give a metric on $S^{p+q+1}$. For this (see [4; p. 14]) we need for every integer $i \geq 1$ and for every $s \geq 0$ :

$$
\begin{aligned}
& \frac{\partial^{(2 i-1)} f}{\partial t^{(2 i-1)}}(s, 0)=0, \\
& f(s, 0)>0, \\
& g^{(2 i)}(0)=0, \\
& g^{\prime}(0)=1, \\
& g(0)=0 ; \\
& \frac{\partial^{(2 i)} f}{\partial t^{(2 i)}}(s, \pi / 2)=0, \\
& \frac{\partial f}{\partial t}(s, \pi / 2)=-1, \\
& f(s, \pi / 2)=0, \\
& g(2 i-1)(\pi / 2)=0, \\
& g(\pi / 2)>0 .
\end{aligned}
$$

Recall from Sect. 3 that we will require our lid to be glued into $S^{n} \times S^{m}-D^{n} \times S^{m}$, where $S^{n} \times S^{m}$ is equipped with a product of round metrics. With this in mind we will demand that for large $s$, our $S^{p+q+1}$ metric will be the unit radius round metric. For this we need $f(s, t)=\cos t$ and $g(t)=\sin t$. Since $g$ is a function of $t$ only, we are therefore led to the following definition.

Definition 6.1 For all $t \in[0, \pi / 2]$, let $g(t)=\sin t$.

In order to perform surgery on some $S^{p} \subset S^{m}$ in the centre of the lid, we will demand that the metric in a neighbourhood of the centre of the lid take the form of a product between a round metric on $S^{p}$ of some (small) radius $\delta$ and some Ricci positive normal metric. As we will outline later, this will allow us to use the Sha-Yang surgery result. 
These metric requirements place restrictions on our choice of function $f$. Precisely, we demand that for sufficiently small $s, f(s, t)$ should be independent of $s$ and satisfy:

(i) $f(s, t)=\delta$ (a constant) for $t \in\left[0, \frac{\pi}{4}\right], s \leq \frac{\epsilon}{10}$, some $\epsilon>0$;

(ii) $f(s, t)=\cos t$ for $t \in\left[\delta^{\prime}, \frac{\pi}{2}\right]$, some $\delta^{\prime}$ very close to $\frac{\pi}{2}, s \leq \frac{\epsilon}{10}$;

(iii) $f_{t t}(s, t) \leq 0$ for all $t \in\left[0, \frac{\pi}{2}\right], s \leq \frac{\epsilon}{10}$;

(iv) $f_{s}(s, t)=0$ for all $t \in\left[0, \frac{\pi}{2}\right], s \leq \frac{\epsilon}{10}$.

The values of $\epsilon, \delta$ and $\delta^{\prime}$ will be fixed later (Definitions 6.4, 6.8 and 6.9).

For values of $s$ between these two extremes, we must show how to define $f$ to obtain a smooth function of both $s$ and $t$ which agrees with the above. With curvature issues in mind, we do this in such a way that

(a) $f(s, t)=\cos t$ for $t \in\left[\delta^{\prime}, \frac{\pi}{2}\right]$ and for all $s$,

(b) $f_{t t}(s, t) \leq 0$ for all $s$ and $t$,

(c) $f_{s}(s, t) \geq 0$ for all $s$ and $t$.

We are now in a position to begin our construction of $f$ and $h$.

Definition 6.2 Let $r_{0} \in\left(0, \frac{\pi}{2}\right)$ be the desired radius of the factor $D^{n}$ in our $\operatorname{lid} D^{n} \times S^{m}$.

\section{Definition 6.3}

(a) The constant $H$ is defined to be $\frac{1}{2}\left(1+\cos r_{0}\right)$.

(b) The constant $H^{\prime}$ is defined to be $\frac{1}{2}(1+H)$.

We will construct the function $h$ so as to have constant slope $H$ at the boundary of the lid. The value of $H$ is chosen so that the $D^{n}$ factor of the lid can be glued within Ricci positivity into its position in the main manifold. (This glueing is discussed in Sect. 7.)

Definition 6.4 The constant $\epsilon \in(0, \pi / 2)$ is fixed at any value for which $\cos \epsilon>H^{\prime}$.

Definition 6.5 The function $h_{0}:[0, \infty) \rightarrow[0, \infty)$ is chosen to be any smooth function satisfying the following requirements:

(i) $h_{0}(s)=\sin s$ for $s \in[0, \epsilon]$;

(ii) $h_{0}^{\prime}(s)=H^{\prime}$ for $s \geq 2 \epsilon$;

(iii) $h_{0}^{\prime \prime}(s) \leq 0$ for all $s \geq 0$.

Observation 6.6 It is clear from the definitions of $h_{0}$ and $g$, and requirement (i) for $f$, that the metric $\mathrm{d} s^{2}+\mathrm{d} t^{2}+f^{2}(s, t) \mathrm{d} s_{p}^{2}+g^{2}(t) \mathrm{d} s_{q}^{2}+h_{0}^{2}(s) \mathrm{d} s_{n-1}^{2}$ must have positve Ricci curvature at least for $s, t \leq \frac{\epsilon}{10}$.

Lemma 6.7 There exists $\kappa=\kappa(p, q, n, \epsilon)>0$ such that surgery can be performed on the copy of $S^{p}$ corresponding to $s=t=0$ preserving the local Ricci positivity, whenever $\delta$, the radius of this $S^{p}$, satisfies $\delta<\kappa$.

Proof For $s, t \leq \frac{\epsilon}{10}$, the metric on the lid is a product between the round metric of radius $\delta$ and a Ricci-positive normal metric. As noted in [5; p. 134] we can deform the metric in normal directions to a round metric of some radius inside a small disk. Notice that the normal metric, and hence the size and curvature of the round disk only depend on $n, q, \epsilon$ and the functions $g(t)=\sin t$ and $h_{0}(s)=\sin s$. By Sha-Yang ([5, Lemma 1]) there exists $\kappa=\kappa(p, q, n, \epsilon)$ such that if $\delta<\kappa$, then we can perform surgery on our $S^{p}$ within Ricci positivity, as claimed. 
Definition 6.8 Fix $\delta$ (see condition (i) for $f$ ) to be any number in the interval $(0, \kappa)$.

Definition 6.9 Fix $\delta^{\prime}$ (see condition (ii) for $f$ ) to be any number in $(0, \pi / 2)$ such that $\cos \delta^{\prime}<\delta$.

We now give more details about the construction of $f$. First some more definitions.

Definition 6.10 Let $f_{1}:[0, \pi / 2] \rightarrow[0,1]$ be given by $f_{1}(t)=\cos t$.

Definition 6.11 Let $f_{2}:[0, \pi / 2] \rightarrow[0,1]$ be any smooth function satisfying

$f_{2}(t)=\delta$ for $t \in[0, \pi / 4]$;

$f_{2}(t)=\cos t$ for $t \in\left[\delta^{\prime}, \pi / 2\right]$;

$f_{2}{ }^{\prime \prime}(t) \leq 0$ for all $t \in[0, \pi / 2]$.

$f_{1}(t)-f_{2}(t)>0$ for all $t \in\left[0, \delta^{\prime}\right)$.

We set

$$
f(s, t)=f_{1}(t) \phi(s)+(1-\phi(s)) f_{2}(t)
$$

with $f_{1}(t)$ and $f_{2}(t)$ as above, and with $\phi(s)$ a smooth 'bump' function satisfying the following:

$\phi(s)=0$ for $s \in[0, \epsilon / 10]$,

$\phi(s)=1$ for all suitably large $s$,

$\phi^{\prime}(s) \geq 0$ for all $s$,

$\phi^{\prime \prime}(s) \geq 0$ for all $s \in[\epsilon / 10, \epsilon]$ and $\phi^{\prime \prime}(s) \leq 0$ for all $s>\epsilon$.

(With curvature issues in mind, note that $f(s, t)$ has the following derivatives:

$$
\begin{aligned}
& f_{s s}(s, t)=\phi^{\prime \prime}(s)\left(f_{1}(t)-f_{2}(t)\right), \\
& f_{s t}(s, t)=\phi^{\prime}(s)\left(f_{1}^{\prime}(t)-f_{2}^{\prime}(t)\right), \\
& \left.f_{t t}(s, t)=\phi(s) f_{1}^{\prime \prime}(t)+(1-\phi(s)) f_{2}^{\prime \prime}(t) .\right)
\end{aligned}
$$

Thus defining $f(s, t)$ boils down essentially to choosing the function $\phi(s)$. Note that because of the choices of $f_{1}$ and $f_{2}$, and the above restrictions of $\phi$, the resulting function $f$ will automatically satisfy all the requirements set out above.

Definition 6.12 Let $\alpha_{0}>0$ be any number satisfying the following conditions:

(i)

$$
\alpha_{0}<\frac{(n-1) \epsilon}{2 p} \cos \delta^{\prime}
$$

(ii)

$$
\alpha_{0}<\frac{n-1}{p} \cos \delta^{\prime} \tan (\epsilon / 10)
$$

(iii)

$$
\alpha_{0}\left[\frac{2}{\epsilon}+(p-1) \alpha_{0}+(n-1) \cot \frac{\epsilon}{10}\right]<(p-1) \cos ^{4} \delta^{\prime}
$$


(iv)

$$
p^{2} \alpha_{0}^{2}+2 p q \cos \delta^{\prime} \frac{\alpha_{0}}{\epsilon}<(n-1) q \cos ^{2} \delta^{\prime}
$$

(It is clear that such a number exists.)

Lemma 6.13 For each $\alpha \in\left(0, \alpha_{0}\right]$ there exists a smooth function $\phi_{1}:[0, \epsilon] \rightarrow[0,1)$ satisfying the following conditions:

(i) $\phi_{1}(s)=0$ for $s \in[0, \epsilon / 10]$;

(ii) $\phi_{1}^{\prime}(\epsilon)=\alpha$;

(iii) $\phi_{1}^{\prime \prime}(s) \in[0,2 \alpha / \epsilon]$ for all $s \in[0, \epsilon]$.

Proof It is clear that we can construct concave up functions with derivative 0 at $s=\epsilon / 10$ and devivative $\alpha$ at $s=\epsilon$, keeping the maximum value of the second derivative arbitrarily close to $10 \alpha / 9 \epsilon$. In particular it is possible to construct such functions keeping the maximum value of the second derivative less than $2 \alpha / \epsilon$.

Proposition 6.14 The metric

$$
\mathrm{d} s^{2}+\mathrm{d} t^{2}+\left[f_{1}(t) \phi_{1}(s)+\left(1-\phi_{1}(s)\right) f_{2}(t)\right]^{2} \mathrm{~d} s_{p}^{2}+g^{2}(t) \mathrm{d} s_{q}^{2}+h_{0}^{2}(s) \mathrm{d} s_{n-1}^{2}
$$

on

$$
[0, \epsilon] \times\left[0, \frac{\pi}{2}\right] \times S^{p} \times S^{q} \times S^{n-1}
$$

has strictly positive Ricci curvature.

Proof We will check each of the curvature formulas (see Sect. 5) in turn, splitting our considerations into the cases $t \geq \delta^{\prime}$ and $t<\delta^{\prime}$.

For notational simplicity, let us write $\theta(s, t)=f_{1}(t) \phi_{1}(s)+\left(1-\phi_{1}(s)\right) f_{2}(t)$.

First we consider the term

$$
\operatorname{Ric}\left(\frac{\partial}{\partial s}, \frac{\partial}{\partial s}\right)=-p \frac{\phi_{1}^{\prime \prime}(s)}{\theta(s, t)}\left(f_{1}(t)-f_{2}(t)\right)-(n-1) \frac{h_{0}^{\prime \prime}(s)}{h_{0}(s)} .
$$

Note that $1>f_{1}(t)-f_{2}(t) \geq 0$ for all $t$. Since $h_{0}(s)=\sin s$ for $s \in[0, \epsilon]$ this formula reduces to

$$
\operatorname{Ric}\left(\frac{\partial}{\partial s}, \frac{\partial}{\partial s}\right)=-p \frac{\phi_{1}^{\prime \prime}(s)}{\theta(s, t)}\left(f_{1}(t)-f_{2}(t)\right)+(n-1) .
$$

The problem here is that $\phi^{\prime \prime} \geq 0$, together with the fact that $\theta(s, t) \rightarrow 0$ as $t \rightarrow \frac{\pi}{2}$.

For $t \geq \delta^{\prime}$ we have $f_{1}(t)=f_{2}(t)$, and hence the curvature term reduces to

$$
\operatorname{Ric}\left(\frac{\partial}{\partial s}, \frac{\partial}{\partial s}\right)=n-1 .
$$

For $t<\delta^{\prime}$ we have $\theta(s, t)>\cos \delta^{\prime}$. It is clear that provided we demand

$$
\phi_{1}^{\prime \prime}(s)<\frac{(n-1)}{p} \cos \delta^{\prime} .
$$

Ricci positivity will be achieved. But this is an immediate consequence of condition (iii) in the definition of $\phi_{1}$ (Lemma 6.13) and condition (i) in Definition 6.12. 
As $g(t)=\sin t$ and $\theta_{t t} \leq 0$, it is clear that the term

$$
\operatorname{Ric}\left(\frac{\partial}{\partial t}, \frac{\partial}{\partial t}\right)>0
$$

for all $s$ and $t$.

Let us now consider the 'mixed' Ricci curvature term $\operatorname{Ric}\left(\frac{\partial}{\partial s}, \frac{\partial}{\partial t}\right)$. As shown above, for $s \in[0, \epsilon]$ we know that the Ricci curvature in both $\partial / \partial s$ and $\partial / \partial t$ directions is strictly positive. From elementary linear algebra, we know that the mixed curvature term will not upset positive Ricci curvature provided

$$
\operatorname{Ric}\left(\frac{\partial}{\partial s}, \frac{\partial}{\partial s}\right) \operatorname{Ric}\left(\frac{\partial}{\partial t}, \frac{\partial}{\partial t}\right)>\left(\operatorname{Ric}\left(\frac{\partial}{\partial s}, \frac{\partial}{\partial t}\right)\right)^{2} ;
$$

or more explicitly:

$$
\begin{aligned}
& \left(-p \frac{\phi_{1}^{\prime \prime}(s)}{\theta(s, t)}\left(f_{1}(t)-f_{2}(t)\right)-(n-1) \frac{h_{0}^{\prime \prime}(s)}{h_{0}(s)}\right)\left(-p \frac{\theta_{t t}(s, t)}{\theta(s, t)}+q\right) \\
& >p^{2}\left(\frac{\phi_{1}^{\prime}(s)}{\theta(s, t)}\right)^{2}\left(f_{1}^{\prime}(t)-f_{2}^{\prime}(t)\right)^{2} .
\end{aligned}
$$

First note that for $t \geq \delta^{\prime}$ and for all $s$ we have $\theta_{s t}(s, t)=0$, and so our inequality ( $\dagger$ ) is automatically true.

For $t<\delta^{\prime}$ is clearly suffices to check that

$$
\left[-\frac{p}{\cos \delta^{\prime}} \sup \phi_{1}^{\prime \prime}+n-1\right] q>p^{2} \frac{\phi_{1}^{\prime}(\epsilon)^{2}}{\cos ^{2} \delta^{\prime}},
$$

which will certainly be the case if

$$
p^{2} \alpha^{2}+p q \cos \delta^{\prime} \sup \phi_{1}^{\prime \prime}<(n-1) q \cos ^{2} \delta^{\prime} .
$$

But this follows from condition (iii) in the definition of $\phi_{1}$ (Lemma 6.13) together with condition (iv) in Definition 6.12.

We now consider the $\operatorname{Ric}\left(W_{k}, W_{k}\right)$ term. Recall that

$$
\operatorname{Ric}\left(W_{k}, W_{k}\right)=-\frac{h_{0}^{\prime \prime}}{h_{0}}+(n-2) \frac{1-\left(h_{0}^{\prime}\right)^{2}}{h_{0}^{2}}-p \frac{\theta_{s} h_{0}^{\prime}}{\theta h_{0}} .
$$

First note that the term $-p \frac{\theta_{s} h_{0}^{\prime}}{\theta h_{0}}$ is non-positive. Now $\theta_{s}(s, t)=\phi_{1}{ }^{\prime}(s)\left(f_{1}(t)-f_{2}(t)\right)$, and as $f_{1}(t)=f_{2}(t)$ for $t \in\left[\delta^{\prime}, \frac{\pi}{2}\right]$, and $\phi_{1}(s)=0$ for $s \in[0, \epsilon / 10], \theta_{s}$ is identically zero for $s$ close to 0 and $t$ close to $\pi / 2$. Therefore this 'bad' term cannot blow up.

As $h_{0}(s)=\sin s$ for $s \in[0, \epsilon]$, we have $-\frac{h_{0}^{\prime \prime}}{h_{0}}=\frac{1-\left(h_{0}^{\prime}\right)^{2}}{h_{0}^{2}}=1$. We therefore need to guarantee that

$$
n-1>p \frac{\left(f_{1}(t)-f_{2}(t)\right) \phi_{1}^{\prime}(s) \cot s}{\theta} .
$$

As noted above, $\phi_{1}^{\prime}(s)=0$ for $s \in[0, \epsilon / 10]$, and $f_{1}(t)-f_{2}(t)=0$ for $t \in\left[\delta^{\prime}, \pi / 2\right]$. It suffices therefore to check that

$$
n-1>\frac{p \cot (\epsilon / 10)}{\cos \delta^{\prime}} \sup _{s \in[0, \epsilon]} \phi_{1}^{\prime}(s) .
$$


Since $\phi_{1}{ }^{\prime} \geq 0$ and $\phi_{1}{ }^{\prime}(\epsilon)=\alpha$, this is equivalent to requiring

$$
\alpha<\frac{n-1}{p} \cos \delta^{\prime} \tan (\epsilon / 10),
$$

which is guaranteed by condition (ii) in Definition 6.12 .

We now come to the $\operatorname{Ric}\left(X_{i}, X_{i}\right)$ term. It is easy to see that when $t \geq \delta^{\prime}, \operatorname{Ric}\left(X_{i}, X_{i}\right)=$ $p+q$. Let us therefore consider $t<\delta^{\prime}$. Note that the term $-q \frac{\theta_{t} g_{t}}{\theta g}$ is non-negative.

As $1 \geq \theta \geq \cos \delta^{\prime}$ and $\theta_{s}=0$ for $s \in\left[0, \frac{\epsilon}{10}\right]$, the 'bad' curvature terms $-\frac{\theta_{s s}}{\theta}$, $-(p-1) \frac{\theta_{s}^{2}}{\theta^{2}}$ and $-(n-1) \frac{\theta_{s} h^{\prime}}{\theta h}$ are at worst

$$
-\frac{\sup _{s \in[0, \epsilon]} \phi_{1}^{\prime \prime}}{\cos \delta^{\prime}}, \quad-(p-1) \frac{\phi_{1}^{\prime}(\epsilon)^{2}}{\cos ^{2} \delta^{\prime}} \quad \text { and }-\frac{(n-1) \phi_{1}^{\prime}(\epsilon)}{\cos \delta^{\prime}} \cot \frac{\epsilon}{10}
$$

respectively. To guarantee Ricci positivity it suffices to check that

$$
\frac{(p-1)}{\theta^{2}}\left(1-\theta_{t}^{2}\right)>\frac{1}{\cos \delta^{\prime}} \sup \phi_{1}^{\prime \prime}+(p-1) \frac{\left(\phi_{1}^{\prime}(\epsilon)\right)^{2}}{\cos ^{2} \delta^{\prime}}+\frac{(n-1) \phi_{1}^{\prime}(\epsilon)}{\cos \delta^{\prime}} \cot \frac{\epsilon}{10} .
$$

Since

$$
\frac{(p-1)}{\theta^{2}}\left(1-\theta_{t}^{2}\right)>(p-1) \cos ^{2} \delta^{\prime}
$$

it therefore suffices to check that

$$
(p-1) \cos ^{2} \delta^{\prime}>\frac{1}{\cos \delta^{\prime}} \sup \phi_{1}^{\prime \prime}+(p-1) \frac{\alpha^{2}}{\cos ^{2} \delta^{\prime}}+\frac{(n-1) \alpha}{\cos \delta^{\prime}} \cot \frac{\epsilon}{10},
$$

which is certainly the case if

$$
\alpha\left[\frac{2}{\epsilon}+(p-1) \alpha+(n-1) \cot \frac{\epsilon}{10}\right]<(p-1) \cos ^{4} \delta^{\prime} .
$$

But this is guaranteed by condition (iii) in Definition 6.12 .

The final curvature expression we have to consider is

$$
\operatorname{Ric}\left(V_{j}, V_{j}\right)=-\frac{g_{s s}+g_{t t}}{g}+\frac{q-1}{g^{2}}\left(1-g_{s}^{2}-g_{t}^{2}\right)-p \frac{\theta_{t} g_{t}}{\theta g} .
$$

It is clear that the first two terms in this are strictly positive, and since $\theta_{t} \leq 0$ the final term is non-negative. Hence $\operatorname{Ric}\left(V_{j}, V_{j}\right)>0$ for all $s$ and $t$. This concludes the proof of Proposition 6.14.

\section{Lemma 6.15}

$$
\sup _{t \in\left[0, \delta^{\prime}\right)}\left[\frac{\left(f_{1}^{\prime}(t)-f_{2}^{\prime}(t)\right)^{2}}{f_{1}(t)-f_{2}(t)}\right]<\infty .
$$

Proof First note that by the last condition in the definition of $f_{2}$ (Definition 6.11), we have that

$$
\frac{\left(f_{1}^{\prime}(t)-f_{2}^{\prime}(t)\right)^{2}}{f_{1}(t)-f_{2}(t)}
$$


is defined for all $t<\delta^{\prime}$. It then clearly suffices to check that

$$
\lim _{t \rightarrow \delta_{-}^{\prime}} \frac{\left(f_{1}^{\prime}(t)-f_{2}^{\prime}(t)\right)^{2}}{f_{1}(t)-f_{2}(t)}<\infty .
$$

By l'Hôpital's Rule, this limit is equal to $\lim _{t \rightarrow \delta_{-}^{\prime}} 2\left(f_{1}^{\prime \prime}(t)-f_{2}^{\prime \prime}(t)\right)$, and clearly this latter limit is 0 .

Definition 6.16 Fix $\eta>1$ to be any number satisfying the following four requirements: (i)

$$
\eta>\frac{p}{q \cos \delta^{\prime}} \sup _{t \in\left[0, \delta^{\prime}\right)}\left[\frac{\left(f_{1}^{\prime}(t)-f_{2}^{\prime}(t)\right)^{2}}{f_{1}(t)-f_{2}(t)}\right]
$$

(ii)

$$
\eta>\frac{p}{(n-2) \cos \delta^{\prime} H^{\prime} \sin ^{2} \epsilon}
$$

(iii)

$$
\eta>\frac{1}{\epsilon^{2} \cos \delta^{\prime}}\left[1+\frac{n-1}{(p-1) H^{\prime} \cos \delta^{\prime}}\right]
$$

(iv)

$$
\eta>\frac{1}{\epsilon \alpha_{0}} .
$$

Definition 6.17 For any given choice of $\phi_{1}$, let $\phi_{2}:[\epsilon, \infty) \rightarrow(0, \infty)$ be given by

$$
\phi_{2}(s)=\phi_{1}(\epsilon)+\frac{1}{\eta} \ln \frac{s}{\epsilon} .
$$

Note that for all $s \geq \epsilon, \phi^{\prime \prime}(s)<0$ and $\phi^{\prime}(s)>0$.

Lemma 6.18 There exists $s_{0}>0$ such that $\phi_{2}\left(s_{0}\right)=1$.

Proof This is immediate since $\phi_{2}(\epsilon)=\phi_{1}(\epsilon)<1$ and $\lim _{s \rightarrow \infty} \phi_{2}(s)=\infty$.

Proposition 6.19 The metric

$$
\mathrm{d} s^{2}+\mathrm{d} t^{2}+\left[f_{1}(t) \phi_{2}(s)+\left(1-\phi_{2}(s)\right) f_{2}(t)\right]^{2} \mathrm{~d} s_{p}^{2}+g^{2}(t) \mathrm{d} s_{q}^{2}+h_{0}^{2}(s) \mathrm{d} s_{n-1}^{2}
$$

on

$$
\left[\epsilon, s_{0}\right] \times\left[0, \frac{\pi}{2}\right] \times S^{p} \times S^{q} \times S^{n-1}
$$

has non-negative Ricci curvature, and strictly positive Ricci curvature for $s$ suitably close to $\epsilon$.

Proof For notational simplicity we set $\psi(s, t)=f_{1}(t) \phi_{2}(s)+\left(1-\phi_{2}(s)\right) f_{2}(t)$. We check each curvature formula in turn.

It is immediate that we have

$$
\operatorname{Ric}\left(\frac{\partial}{\partial s}, \frac{\partial}{\partial s}\right) \geq 0 .
$$


Note that the Ricci curvature in this direction is zero for $s \geq 2 \epsilon\left(\right.$ when $\left.h^{\prime \prime}(s)=0\right)$ and $t \geq \delta^{\prime}\left(\right.$ when $\left.f_{1}(t)-f_{2}(t)=0\right)$. However, since $h^{\prime \prime}(\epsilon)=-\sin (\epsilon)$ and $\phi_{2}^{\prime \prime}(\epsilon)=-\eta^{-1} \epsilon^{-2}$ we certainly have strictly positive Ricci curvature for $s$ close to $\epsilon$.

It is clear that we have

$$
\operatorname{Ric}\left(\frac{\partial}{\partial t}, \frac{\partial}{\partial t}\right)>0
$$

for all $s \geq \epsilon$ and $t \in[0, \pi / 2]$.

We now consider the mixed Ricci curvature term. Since the Ricci curvatures in the $\partial / \partial s$ and $\partial / \partial t$ directions are at worst non-negative, the mixed curvature term will not upset Ricci non-negativity provided

$$
\operatorname{Ric}\left(\frac{\partial}{\partial s}, \frac{\partial}{\partial s}\right) \operatorname{Ric}\left(\frac{\partial}{\partial t}, \frac{\partial}{\partial t}\right) \geq\left(\operatorname{Ric}\left(\frac{\partial}{\partial s}, \frac{\partial}{\partial t}\right)\right)^{2} .
$$

For $t \geq \delta^{\prime}$ and for all $s \geq \epsilon$ we have $\psi_{s t}(s, t)=0$, and so $(\ddagger)$ is true. In fact for $t \geq \delta^{\prime}$ and $s$ suitably close to $\epsilon$, strict inequality holds in (†) since Ric $\left(\frac{\partial}{\partial s}, \frac{\partial}{\partial s}\right)>0$ for such values of $s$, as noted above. We obtain equality in ( $\ddagger)$ for $t \geq \delta^{\prime}$ and $s \geq 2 \epsilon$ since $h_{0}{ }^{\prime \prime}$ is zero for these values of $s$.

For $t<\delta^{\prime}$ we obtain strict inequality in ( $\ddagger$ ) provided

$$
-\phi_{2}^{\prime \prime}(s)>\frac{p}{q} \frac{1}{\cos \delta^{\prime}} \frac{\left(f_{1}^{\prime}(t)-f_{2}^{\prime}(t)\right)^{2}}{f_{1}(t)-f_{2}(t)}\left(\phi_{2}^{\prime}(s)\right)^{2} .
$$

Using the definition of $\phi_{2}$, we can reduce this to

$$
\eta>\frac{p}{q} \frac{1}{\cos \delta^{\prime}} \frac{\left(f_{1}^{\prime}(t)-f_{2}^{\prime}(t)\right)^{2}}{f_{1}(t)-f_{2}(t)} .
$$

But this is guaranteed by condition (i) in the definition of $\eta$. Note that since we have Ricci positivity in the $\partial / \partial s$ and $\partial / \partial t$ directions and strict inequality in ( $\ddagger$ ) for $s$ suitably close to $\epsilon$, the mixed curvature term cannot upset Ricci positivity at these values of $s$.

We now come to the term $\operatorname{Ric}\left(W_{k}, W_{k}\right)$ :

$$
\operatorname{Ric}\left(W_{k}, W_{k}\right)=-\frac{h_{0}^{\prime \prime}}{h_{0}}+(n-2) \frac{1-\left(h_{0}^{\prime}\right)^{2}}{h_{0}^{2}}-p \frac{\psi_{s} h_{0}^{\prime}}{\psi h_{0}} .
$$

The first two terms in this expression are non-negative and positive respectively for all $s \geq \epsilon$. For $t \geq \delta^{\prime}$ the third term is identically zero, so overall we have Ricci positivity for these values of $t$.

For $t<\delta^{\prime}$ we can bound $\psi(s, t)$ below by $\cos \delta^{\prime}$. Recall that $\phi_{2}^{\prime}(s)=1 / \eta s$. Also notice that $h_{0}(s)>H^{\prime} s$. We can now make the following estimate:

$$
\frac{\psi_{s} h_{0}^{\prime}}{\psi h_{0}}<\frac{1}{\eta s} \frac{1}{H^{\prime} s} \frac{1}{\cos \delta^{\prime}} .
$$

Now

$$
1-\left(h_{0}^{\prime}\right)^{2} \geq 1-h_{0}^{\prime}(\epsilon)^{2}
$$

and $h_{0}(s)<s$, so

$$
\frac{1-\left(h_{0}^{\prime}\right)^{2}}{h_{0}^{2}} \geq \frac{1-h_{0}^{\prime}(\epsilon)^{2}}{s^{2}} .
$$


Since $h_{0}^{\prime}(\epsilon)=\cos \epsilon$ we can make the following estimate:

$$
\operatorname{Ric}\left(W_{k}, W_{k}\right) \geq(n-2) \frac{\sin ^{2}(\epsilon)}{s^{2}}-\frac{p}{\eta s^{2} H^{\prime} \cos \delta^{\prime}} .
$$

We therefore have strict Ricci positivity provided

$$
\eta>\frac{p}{(n-2) H^{\prime} \cos \delta^{\prime} \sin ^{2} \epsilon} .
$$

But this is just condition (ii) in the definition of $\eta$.

For the $\operatorname{Ric}\left(X_{i}, X_{i}\right)$ term we have $\operatorname{Ric}\left(X_{i}, X_{i}\right)=p+q$ for $t \geq \delta^{\prime}$. For $t<\delta^{\prime}$ we need to note that $\phi_{2}^{\prime \prime}(s)<0$, so $\phi_{2}(s)$ is decreasing with $s, \psi(s, t)$ is bounded above by 1 for $s \in\left[\epsilon, s_{0}\right]$, and $\psi_{t}$ is bounded above by $\sin \delta^{\prime}$ for all $s \geq \epsilon$ and $t<\delta^{\prime}$. We can therefore bound the curvature expression below as follows:

$$
\operatorname{Ric}\left(X_{i}, X_{i}\right)>(p-1)\left[\cos ^{2} \delta^{\prime}-\left(\phi_{2}^{\prime}(\epsilon)\right)^{2}\right]-(n-1) \frac{\phi_{2}^{\prime}(\epsilon)}{\cos \delta^{\prime}} \frac{1}{H^{\prime} \epsilon} .
$$

Strict positivity of the right-hand-side is guaranteed if

$$
\frac{n-1}{H^{\prime} \epsilon \cos \delta^{\prime}} \phi_{2}^{\prime}(\epsilon)+(p-1)\left(\phi_{2}^{\prime}(\epsilon)\right)^{2}<(p-1) \cos ^{2} \delta^{\prime}
$$

But $\phi_{2}^{\prime}(\epsilon)=\frac{1}{\eta \epsilon}$, so this condition is equivalent to

$$
\frac{n-1}{\eta H^{\prime} \epsilon^{2} \cos \delta^{\prime}}+\frac{p-1}{\eta^{2} \epsilon^{2}}<(p-1) \cos ^{2} \delta^{\prime}
$$

Since $\eta>1$ it suffices to check that

$$
\frac{1}{\eta}\left(\frac{n-1}{H^{\prime} \epsilon^{2} \cos \delta^{\prime}}+\frac{p-1}{\epsilon^{2}}\right)<(p-1) \cos \delta^{\prime} .
$$

But this is equivalent to condition (iii) in the definition of $\eta$.

The final curvature term, $\operatorname{Ric}\left(V_{j}, V_{j}\right)$ is easily seen to be strictly positive. This concludes the proof of Proposition 6.19.

Lemma 6.20 There exists $\alpha \in\left(0, \alpha_{0}\right)$ and a corresponding function $\phi_{1}:[0, \epsilon] \rightarrow[0,1)$ satisfying the conditions laid out in Lemma 6.13 such that the function $\phi_{3}:[0, \infty) \rightarrow$ $[0, \infty)$ defined by

$$
\phi_{3}(s)=\left\{\begin{array}{l}
\phi_{1}(s) \text { for } s \leq \epsilon \\
\phi_{2}(s) \text { for } s>\epsilon
\end{array}\right.
$$

is $C^{1}$ at $s=\epsilon$.

Proof By definition of $\phi_{2}$ we see $\phi_{3}$ is $C^{0}$ at $s=\epsilon$ for any choice of $\alpha$ and $\phi_{1}$. Now $\phi_{2}^{\prime}(\epsilon)=\frac{1}{\eta \epsilon}$ and $\phi_{1}^{\prime}(\epsilon)=\alpha$. Since by condition (iv) in the definition of $\eta$ we have $\alpha_{0}>1 / \eta \epsilon$, we are free to choose $\alpha=1 / \eta \epsilon$, making a $C^{1}$ join between $\phi_{1}$ and $\phi_{2}$.

We now fix this value of $\alpha$ and the corresponding functions $\phi_{1}$ and $\phi_{2}$.

Lemma 6.21 It is possible to smooth $\phi_{3}$ to a function $\phi_{4}$ by making an arbitrarily small adjustment in an arbitrarily small neighbourhood of $s=\epsilon$ such that the metric

$$
\mathrm{d} s^{2}+\mathrm{d} t^{2}+\left[f_{1}(t) \phi_{4}(s)+\left(1-\phi_{4}(s)\right) f_{2}(t)\right]^{2} \mathrm{~d} s_{p}^{2}+g^{2}(t) \mathrm{d} s_{q}^{2}+h_{0}^{2}(s) \mathrm{d} s_{n-1}^{2}
$$


has non-negative Ricci curvature on $\left[0, s_{0}\right] \times\left[0, \frac{\pi}{2}\right] \times S^{p} \times S^{q} \times S^{n-1}$, and strictly positive Ricci curvature at least for $s \in[0, \epsilon]$.

Proof Suppose that the smoothing takes place over an interval $\left(\epsilon-\epsilon^{\prime}, \epsilon+\epsilon^{\prime}\right)$ for some $\epsilon^{\prime} \ll \epsilon$. Of course we are free to choose $\epsilon^{\prime}$ as small as we wish, and by Propositions 6.14 and 6.19 we see that provided $\epsilon^{\prime}$ is suitably small, this metric has strictly positive Ricci curvature at both $s=\epsilon-\epsilon^{\prime}$ and $s=\epsilon+\epsilon^{\prime}$. It is clear that we can make our adjustment to $\phi_{3}$ ensuring that $\phi_{4}^{\prime \prime}(s)$ interpolates between the values $\phi_{3}^{\prime \prime}\left(\epsilon-\epsilon^{\prime}\right)$ and $\phi_{3}{ }^{\prime \prime}\left(\epsilon+\epsilon^{\prime}\right)$ for $s \in\left(\epsilon-\epsilon^{\prime}, \epsilon+\epsilon^{\prime}\right)$, and the values of $\phi_{3}$ and $\phi_{3}^{\prime}$ are altered negligibly. Since the only Ricci curvature terms for the above metric involving $\phi_{4}^{\prime \prime}$ depend linearly on $\phi_{4}^{\prime \prime}$, it is clear that the smoothing can be made within Ricci positivity.

Proposition 6.22 There exists a constant $\epsilon^{\prime \prime}>0$ and smooth functions $\phi, h:[0, \infty) \rightarrow$ $[0, \infty)$ such that

$$
\begin{array}{ll}
\text { (i) } & \phi(s)=\phi_{4}(s) \text { for } s \leq s_{0}-\epsilon^{\prime \prime} ; \\
\text { (ii) } \phi^{\prime \prime}(s) \leq 0 \text { for } s \geq s_{0}-\epsilon^{\prime \prime} ; \\
\text { (iii) } \phi(s)=1 \text { for all } s \geq s_{0}+\epsilon^{\prime \prime} ; \\
\text { (iv) } h(s)=h_{0}(s) \text { for } s \leq s_{0}-\epsilon^{\prime \prime} ; \\
\text { (v) } h^{\prime \prime}(s) \leq 0 \text { for all } s \in[0, \infty) ; \\
\text { (vi) } h^{\prime}(s)=H \text { for } s \geq s_{0}+\epsilon^{\prime \prime} ;
\end{array}
$$

and the Ricci curvature of the metric

$$
\mathrm{d} s^{2}+\mathrm{d} t^{2}+f^{2}(s, t) \mathrm{d} s_{p}^{2}+g^{2}(t) \mathrm{d} s_{q}^{2}+h^{2}(s) \mathrm{d} s_{n-1}^{2}
$$

on

$$
[0, \infty) \times\left[0, \frac{\pi}{2}\right] \times S^{p} \times S^{q} \times S^{n-1}
$$

has non-negative Ricci curvature, strictly positive at some points, where as before

$$
f(s, t)=f_{1}(t) \phi(s)+(1-\phi(s)) f_{2}(t) .
$$

Proof Notice first that the existence of functions $\phi$ and $h$ satisfying requirements (i)-(vi) above is trivial. We create the functions $\phi(s)$ and $h(s)$ by performing concavedown bends to $\phi_{4}(s)$ respectively $h_{0}(s)$ over the interval $s \in\left[s_{0}-\epsilon^{\prime \prime}, s_{0}+\epsilon^{\prime \prime}\right]$. Notice that if $\epsilon^{\prime \prime}$ is chosen sufficiently small, these deformations can be made with arbitrarily small change to either the values taken by the functions or their first derivatives on $s \in\left[s_{0}-\epsilon^{\prime \prime}, s_{0}+\epsilon^{\prime \prime}\right]$.

We need to check the Ricci curvature claim. First note that for $s \geq s_{0}+\epsilon^{\prime \prime}$ the Ricci curvature is easily seen to be non-negative, since we have $h^{\prime \prime}(s)=0$ and for all $t \in[0, \pi / 2]$ we have $f_{s s}(s, t)=f_{s t}(s, t)=f_{s}(s, t)=0$. For $s \in\left[s_{0}-\epsilon^{\prime \prime}, s_{0}+\epsilon^{\prime \prime}\right]$ we need to make a more detailed analysis, and as before consider the curvature expressions on a case by case basis.

Ricci non-negativity for the Ric $\left(\frac{\partial}{\partial s}, \frac{\partial}{\partial s}\right)$ term is immediate since $\phi^{\prime \prime}(s) \leq 0$ and $h^{\prime \prime}(s) \leq 0$ when $s \in\left[s_{0}-\epsilon^{\prime \prime}, s_{0}+\epsilon^{\prime \prime}\right]$.

The Ric $\left(\frac{\partial}{\partial t}, \frac{\partial}{\partial t}\right)$ is trivially seen to be strictly positive at these values of $s$.

For the mixed curvature term, we need to give more detail about how $\phi_{4}(s)$ should be deformed to a constant function with value 1 over the interval $\left[s_{0}-\epsilon^{\prime \prime}, s_{0}+\epsilon^{\prime \prime}\right]$ so as not to introduce any negative Ricci curvature. We consider again the inequality ( $)$.

Notice that the size of the mixed derivative $f_{s t}(s, t)=\phi^{\prime}(s)\left(f_{1}^{\prime}(t)-f_{2}^{\prime}(t)\right)$ is bounded above (for example by $2 \alpha$ ). As the slope of $h(s)$ is $H^{\prime}>H$ at the start of the interval, 
we can make a further concave-down bend to $h$, and still keep $h^{\prime}(s)>H$ at the end of the interval. By choosing the interval (in other words $\epsilon^{\prime \prime}$ ) sufficiently small, we can arrange for $-q(n-1) \frac{h_{s s}}{h}$ (a lower bound for the left-hand-side of $(\ddagger)$ ) to dominate the square of the mixed curvature term (which is independent of $h$ ). Within this $h$ deformation interval, deform $\phi_{4}$ to a constant function with value 1 in a concave-down manner. As such a deformation decreases the value of the squared mixed curvature term, and does not affect $-q(n-1) \frac{h_{s s}}{h}$, it is clear that $\left.\ddagger\right)$ holds throughout. Finally, with a further concave-down deformation if necessary, ensure that $h$ has (constant) slope $H$. This final deformation clearly cannot cause ( $\ddagger)$ to be violated.

The term $\operatorname{Ric}\left(W_{k}, W_{k}\right)$ will be strictly positive for $s \in\left[s_{0}-\epsilon^{\prime \prime}, s_{0}+\epsilon^{\prime \prime}\right]$, provided $\epsilon^{\prime \prime}$ is chosen suitably small. To see this note that both $f_{s}$ and $h^{\prime}$ decrease over this interval (fixed $t$ ), but the corresponding change in $f h$ can be assumed to be negligible, simply by choosing the interval small enough. Thus $(n-2) \frac{1-h^{\prime}(s)^{2}}{h(s)^{2}}$, say, will still dominte the $-p \frac{f_{s} h^{\prime}}{f h}$ term.

The lower bound given for $\operatorname{Ric}\left(X_{i}, X_{i}\right)$ in Proposition 6.19 is still valid for $s \in\left[s_{0}-\epsilon^{\prime \prime}, s_{0}+\epsilon^{\prime \prime}\right]$, so we see the Ricci curvature in this direction is strictly positive for these values of $s$.

Finally, $\operatorname{Ric}\left(V_{j}, V_{j}\right)$ is trivially seen to be strictly positive. This concludes the proof of Proposition 6.22.

Observation 6.23 The functions $f, g$ and $h$ in Proposition 6.22 satisfy (by construction) all the boundary conditions detailed at the start of Sect. 6 . The function $f$ satisfies the additional requirements (i)-(iv) and (a)-(c) laid out after Definition 6.1. As a consequence of Lemma 6.7 and the choice of $\delta$ (Definition 6.8) we can perform surgery within Ricci positivity on the copy of $S^{p}$ corresponding to $s=t=0$.

Note that we can arrange for the boundary of the lid to correspond to $s=s_{1}$, for any $s_{1}>s_{0}+\epsilon^{\prime \prime}$. Of course we require the radius of the $D^{n}$ factor of the lid to be $r_{0}$ (see Definition 6.2). This can be achieved by performing a global rescale of the lid. Such a rescale does not affect the Ricci curvature. This completes our lid metric construction.

\section{Proofs of the main theorems}

Proof of Theorem A As noted in Sect. 2, we can form $\left(S^{n} \times S^{m}\right) \sharp\left(S^{p+1} \times S^{n+m-p-1}\right)$ by performing surgery on $\{*\} \times S^{p} \subset S^{n} \times S^{m}$, assuming of course that $p<m$.

As noted at the start of Sect. 3, we assume that $S^{n} \times S^{m}$ is equipped with a product of round metrics: $S^{n}$ of unit radius, and the radius of $S^{m}$ to be determined later.

We remove a copy of $D_{r_{0}}^{n}(1) \times S^{m}$ from $S^{n} \times S^{m}$ for some $r_{0} \in(0, \pi / 2)$, and wish to replace it with a 'lid', as constructed in Sect. 6.

For $s>s_{0}+\epsilon$, the outer 'annulus' of our lid is metrically

$$
\left(I \times S^{n-1} \times S^{p+q+1} ; \mathrm{d} s^{2}+h^{2}(s) \mathrm{d} s_{n-1}^{2}+\mathrm{d} s_{p+q+1}^{2}\right)
$$

for some $s$-interval $I$. The Ricci curvature of this is non-negative. As zero curvatures arise precisely when $h^{\prime \prime}(s)=0$, we see that any concave-down bend of $h$ will give Ricci positivity during the bending.

The disk $D^{n}$ clearly fits into a round hole in $S^{n}(1)$ to give a $C^{0}$ join after rescaling. Since $h^{\prime}(s)=H$ and $h^{\prime \prime}(s)=0$ at the boundary of the lid, a suitable concave-down 
bending of $h$ in some arbitrarily small neighbourhood of the lid boundary will result in a smooth join (after rescaling) without introducing any negative Ricci curvatures.

Notice that a global rescale of the lid affects neither the Ricci curvature, nor $h^{\prime}(s)$ (both $s$ and $h(s)$-axes are rescaled). Suppose that we require a metric rescaling factor of $\beta^{2}$ in order for $D^{n}$ to fit. Then the whole lid can be glued into the hole in $S^{n} \times S^{m}$ if we equip the latter with the product metric $\mathrm{d} s_{n}^{2}+\beta^{2} \mathrm{~d} s_{m}^{2}$.

As noted in Observation 6.23, a locally Ricci positive $p$-surgery can be performed at the centre of the lid, yielding a Ricci non-negative metric on $\left(S^{n} \times S^{m}\right) \sharp\left(S^{p+1} \times\right.$ $S^{n+m-p-1}$ ) with strict Ricci positivity at many points. By [2] this metric can be deformed to one with everywhere strictly positive Ricci curvature.

Suppose now we want to perform multiple surgeries and therefore have different lids. In general these will have different values for $h(s)$ at their boundaries. Recall that we assume the holes into which we want the lids to fit have disks of identical radii. Choose the lid with the biggest $h(s)$ at its boundary. Extend the range of $s$ for all the other lids so their $h(s)$ values agree with the largest. There is no problem with doing this, metrically or otherwise. After these extensions, a single lid rescaling factor will work for all lids, and therefore we can fix a metric $S^{n}(1) \times S^{m}(\beta)$ on the ambient manifold.

As before, we want to perform surgery on a sphere at the centre of each of these lids, and obtain a Ricci positive metric on a connected sum involving several terms. It remains to establish that all connected sums of products of spheres, where each individual sphere has dimension at least 3 , can be obtained in this way.

First note that if $n=m=3$, the only possible connected sums satisfying the dimension requirements of Theorem $\mathrm{A}$ are those taking the form

$$
S^{3} \times S^{3} \sharp \cdots \sharp S^{3} \times S^{3} .
$$

These manifolds admit positive Ricci curvature metrics by Sha and Yang.

Let us now assume (without loss of generality) that $m \geq n$. Since we have dealt with the $n=m=3$ case, we can assume also that $m \geq 4$. As observed at the start of Sect. 6, we can perform local Ricci positive surgeries of dimensions $2,3, \ldots,(m-2)$ on the $S^{m}$ factor. One such surgery can therefore yield any of the following manifolds: $\left(S^{n} \times S^{m}\right) \sharp\left(S^{3} \times S^{n+m-3}\right),\left(S^{n} \times S^{m}\right) \sharp\left(S^{4} \times S^{n+m-4}\right), \ldots,\left(S^{n} \times S^{m}\right) \sharp\left(S^{m-1} \times S^{n+1}\right)$. Thus we can produce Ricci positive connected sums with products involving $S^{3}, S^{4}, \ldots, S^{m-1}$ and simultaneously $S^{n+1}, \ldots, S^{n+m-3}$. If $m>n$, we see that all factor sphere dimensions $S^{3}, \ldots, S^{n+m-3}$ are covered. However in any connected sum between products of spheres (except $\left(S^{n} \times S^{n}\right) \sharp \cdots \sharp\left(S^{n} \times S^{n}\right)$ which admits a Ricci positive metric by Sha-Yang) there must be a product $S^{n} \times S^{m}$ with $m>n$. By performing surgery on such a product, we can clearly obtain all the desired examples.

Proof of Theorem $B$ We can construct a submersion metric with totally geodesic fibres on $M$ by specifying a metric on $X$, a metric on $S^{m}$, and a connection on the associated principal bundle, (see [1; p. 249] for details). Given any Ricci positive metric on $X$ and any choice of connection, there is a $\beta>0$ such that choosing the round metric of radius $\beta$ on $S^{m}$ will produce a Ricci positive metric on $M$. See [1; p. 253]. We are free to choose the connection so that it is flat over some disjoint neighbourhoods $U_{1}, \ldots, U_{k}$ of $B$. This means that the corresponding submersion metric will locally be a product over these regions. As noted earlier, we are also free to locally deform the metric on $X$ within each of these neighbourhoods so that we get a round metric of radius $N_{i}$ in a smaller neighbourhood of some radius $R_{i}$, without introducing any 
non-positive Ricci curvatures. In our construction to establish Theorem A, we assumed that all disks chosen on $S^{n}$ were of the same size $r_{0}$. We can arrange for that again here by setting $r_{0}=\inf _{i} R_{i}$, and restricting our attention to disks of this size. By setting $H=(1 / 2)\left[1+\sup _{i} \cos \left(r_{0} / N_{i}\right)\right]$ (see Definition 6.3), our lid construction and glueing procedure can be applied simultaneously to all neighbourhoods, provided $\beta$ is chosen small enough. Surgery can then be performed to obtain the required examples. Note that to obtain all the connected sums claimed we require $m>n$. This follows from the argument given in the last paragraph of the proof of Theorem A.

Proof of Theorem $C$ First note that any sphere bundle with base $S^{p+1}$, fibre $S^{n+m-p-1}$, and structural group $S O(n+m-p)$, can be constructed from two copies of $D^{p+1} \times$ $S^{n+m-p-1}$ by gluing along their boundaries according to a smooth map $T: S^{p} \rightarrow$ $S O(n+m-p)$. Such a map $T$ also specifies a trivialisation of the normal bundle of $S^{p}$ in our lid. (Note that this normal bundle has a canonical trivialisation preserving the product metric structure. Any other trivialisation can be obtained from this one by 'twisting' it using a suitable map T.) It is easy to see that performing surgery on $S^{p}$ using the trivialisation determined by $T$ results in a connected sum with the $S^{n+m-p-1}$ - bundle over $S^{p+1}$ determined by $T$. The Theorem follows easily from Theorem B if we replace the Sha-Yang surgery result by Theorem 0.3 of [6], which allows us to handle other trivialisations of the normal bundle. However, to use this result we need the dimension of the surgery $>$ codimension $\geq 3$. In other words we need $p>n+q+1$. Thus we obtain the extra requirements $p \geq n+3$ and $m \geq n+5$, which result in the dimension restrictions for $E_{1}, \ldots, E_{k}$.

\section{References}

1. Besse, A.: Einstein manifolds. Springer-Verlag (1987)

2. Ehrlich, P.: Metric deformations of curvature. Geom. Dedicata 5, 1-23 (1976)

3. Gromov, M., Lawson, H.B.: The classification of manifolds of positive scalar curvature. Ann. Math. 111, 423-434 (1980)

4. Petersen, P.: Riemannian Geometry, Graduate Texts in Mathematics, Vol. 171. SpringerVerlag (1997)

5. Sha, J.-P., Yang, D.-G.: Positive Ricci curvature on connected sums of $S^{n} \times S^{m}$. J. Diff. Geom. 33, 127-138 (1990)

6. Wraith, D.J.: Surgery on Ricci positive manifolds. J. Reine Angew. Math. 501, 99-113 (1998) 